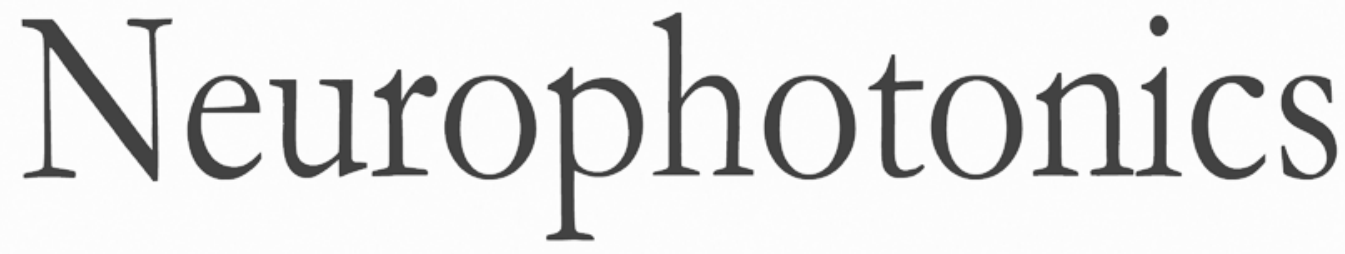

\title{
Using a simulation approach to optimize time-domain diffuse correlation spectroscopy measurement on human head
}

Lina Qiu

Huiyi Cheng

Alessandro Torricelli

Jun Li 


\title{
Using a simulation approach to optimize time-domain diffuse correlation spectroscopy measurement on human head
}

\author{
Lina Qiu, ${ }^{a}$ Huiyi Cheng, ${ }^{b}$ Alessandro Torricelli, ${ }^{a}, \mathrm{c}$ and Jun $\mathrm{Li}^{\mathrm{b}, *}$ \\ ${ }^{a}$ Dipartimento di Fisica, Politecnico di Milano, Milan, Italy \\ 'South China Normal University, Guangdong Provincial Key Laboratory of Optical Information Materials and Technology, National Center for \\ International Research on Green Optoelectronics, MOE International Laboratory for Optical Information Technologies, South China Academy of \\ Advanced Optoelectronics, Guangzhou, China \\ ${ }^{\circ}$ Consiglio Nazionale delle Ricerche, Istituto di Fotonica e Nanotecnologie, Milan, Italy
}

\begin{abstract}
Time-domain diffuse correlation spectroscopy (TD-DCS) has been recently proposed to improve detection of deep blood flow dynamics in a biological tissue, such as human brain. To obtain a high sensitive measurement, several experimental parameters such as the source-detector (SD) distance, gate opening time, and width need to be considered and optimized. We use a simulation approach to optimize these parameters based on Monte Carlo computations using a realistic human head model. Two cortical regions are investigated including the frontal and temporal lobes. SD distance ranging from 0 to $45 \mathrm{~mm}$, gate opening time from 400 to $1000 \mathrm{ps}$, and gate width from 50 to $3000 \mathrm{ps}$ are considered. The goal is to find out the optimal combinations of these parameters by which the higher contrast measurement on the cortical dynamics can be achieved. The simulations show that with an acceptable input power of light, the combinations of SD distance ranging from 0 to $15 \mathrm{~mm}$, gate opening time at 700 to $800 \mathrm{ps}$, and gate width of $800 \mathrm{ps}$ are optimal for achieving higher contrast measurement on the cortical dynamics. The simulation approach and results are helpful for the optimization of TD-DCS experimental design focused on brain functional detection. ๑ 2018 Society of Photo-Optical Instrumentation Engineers (SPIE) [DOI: 10.1117/1.NPh.5.2.025007]
\end{abstract}

Keywords: diffuse correlation spectroscopy; time-domain; simulation; optimization; Monte Carlo; brain functional detection. Paper 17143RR received Dec. 30, 2017; accepted for publication Apr. 16, 2018; published online May 14, 2018.

\section{Introduction}

Diffuse correlation spectroscopy (DCS) is a rapidly growing optical technique, enabling noninvasive measurements of the dynamical properties of a scattering medium such as biological tissue. ${ }^{1-6}$ When tissue is illuminated by a laser beam with long coherence length (e.g., much longer than the typical path length in the tissue), the emitted light intensity pattern on the surface is composed of many bright and dark spots called speckles. ${ }^{5}$ If the scattering particles are moving, the speckle pattern will fluctuate in time. DCS exploits the coherent light to study speckle fluctuations arising from multiple scattering in the tissue. In living tissue, light scattering from red blood cells causes the temporal fluctuations of speckle pattern, which are typically quantified by measuring the temporal autocorrelation function $\left(g_{2}\right)$ of the detected light intensity. By measuring the intensity fluctuation, it is possible to obtain information about the motion of the scattering particles, such as blood flow. The measured $g_{2}$ is a decay curve with respect to the lag time, which contains rich information about the motion of the scatterers. In general, faster decay indicates faster dynamics. ${ }^{5,7}$

DCS is originally performed with a continuous-wave $(\mathrm{CW})$ laser source with a coherent length much longer than the pathlengths of the detected photons to ensure interference of light traveling along different paths. ${ }^{5,8}$ However, CW-DCS does not readily discriminate between contributions of photons

*Address all correspondence to: Jun Li, E-mail: jun.li@coer-scnu.org coming from shallow and deep layers, because both short and long pathlengths contribute to the detected light and $g_{2}$. In addition, CW-DCS is sensitive to superficial cortical regions, lacking the ability to detect the deep dynamics. By enlarging the source-detector (SD) distance, the penetration depth can be increased in CW-DCS, but the detected light intensity dramatically decreases at the same time. In particular, DCS typically employs single-mode detection fibers for measurement, which easily leads to higher noise level on $g_{2}$. Although one could use higher input light power to improve the signal quality of $g_{2}$, however, due to safety reason for human tissue, there is an upper limit for the incident power that has to be taken into account when considering the sensitivity of DCS measurement to the brain. As reported in the ANSI Standard Z136.1-1993 Table, ${ }^{9}$ the maximum permissible exposure of skin to laser radiation is between 2.6 and $4 \mathrm{~mW} / \mathrm{mm}^{2}$ in 760 to $850 \mathrm{~nm}$.

Recently, an implementation and theory for time-domain DCS (TD-DCS), or pathlength-resolved DCS, has been developed, aiming at improving the depth sensitivity by discriminating short and long photon pathlengths. ${ }^{10-12}$ By employing a pulsed laser with a certain coherence length, Sutin et al. ${ }^{10}$ simultaneously measured the time-of-flight of photons and DCS speckle fluctuations and demonstrated that TD-DCS was able to separate the contribution of photons from shallow and deep layers. Very recently this technique has also been successfully applied to in vivo measurements on human tissues 
including adult head. ${ }^{11}$ At the same time, TD-DCS analytical theory for multilayer and heterogeneous turbid media was also presented. ${ }^{12}$ The photon detection of TD-DCS is very similar to time-resolved spectroscopy (TRS) system, except for the detection fibers. TD-DCS uses single mode fiber to ensure detection of light from a single speckle (or a single coherence area). TRS usually utilizes fiber bundles to collect the emitted light, thus the intensity of detected light is much higher than that of DCS. Apart from that, both techniques are based on the wellestablished time gate method by which the time-of-flight of each detected photon is defined. In this paper, we call the time when the gate is ready to record photon the gate opening time. Another striking difference between TD-DCS and TRS is the source: although both of them need pulsed source, TD-DCS requires coherent light with a certain coherence length (e.g., at least several centimeters), whereas TRS has no such requirement.

In contrast to the traditional CW-DCS, TD-DCS can timegate the signals for only late arriving photons, which greatly improve the sensitivity of detection to the deeper dynamics in a scattering sample such as the human brain. CW-DCS measures the dynamics sampled by photons with a wide range of path lengths, thus has little ability to differentiate between deeper and superficial dynamics. For TD-DCS, it becomes feasible to probe the dynamics sampled by photons with a single pathlength (or a very narrow distribution of the pathlengths). Therefore, TD-DCS is able to provide pathlength-resolved measurement on dynamics property of tissue. However, to obtain a high sensitive cortical dynamics measurement with TD-DCS under a limited input laser power, several experiment-related parameters such as the gate opening time, gate width, and SD distance need to be considered. In this study, we used a simulation approach to optimize these experimentrelated parameters based on Monte Carlo (MC) computations using a realistic human-head model $\left(\right.$ Collin27) ${ }^{13}$ in order to achieve higher contrast measurement on the dynamics [e.g., cerebral blood flow (CBF)] change in deep (cortical) layer of human head. Two cortical regions were studied including the frontal and temporal lobes. The aim of this work is to find out the optimal combinations of SD distance, gate opening time, and width by which the highest contrast measurement on the change of cortical dynamics can be achieved.

\section{Methods}

\subsection{Theory}

The light intensity autocorrelation function $\left(g_{2}\right)$ was considered in this study, because $g_{2}$ is recorded instead of the normalized electric field autocorrelation function $\left(g_{1}\right)$ in real measurements. $g_{2}$ can be derived from $g_{1}$ via the Siegert relation, $g_{2}=1+\beta\left|g_{1}\right|^{2} .{ }^{14}$ Here, $\beta$ is a parameter (the intercept of $g_{2}$ at zero correlation lag time) depending on the laser coherence length and on the number of modes in the detection fiber. If the coherence length is long enough, photon detection with a single mode fiber gives rise to $\beta=0.5$ for the unpolarized light. For simplicity, we only considered Brownian motion as dynamics in the sample. It is because that many studies have demonstrated Brownian motion model can better explain the experimental data measured from living tissues including the human brain. ${ }^{7,8,15}$ Then, the theoretical model for pathlength-resolved $g_{1}$ can be described as ${ }^{10}$

$g_{1}(\tau, s)=\exp \left(-2 k^{2} \mu_{s}^{\prime} s D_{B} \tau\right)$ where $\tau$ is the correlation lag time, $s$ is the pathlength, $k$ is the wave number in the medium, $\mu_{\mathrm{s}}^{\prime}$ is the reduced scattering coefficient, and $D_{B}$ is the Brownian diffusion coefficient of the scattering particles. For pathlength ranging from $s_{0}-\Delta s$ to $s_{0}+\Delta s$, we have

$g_{1}\left(\tau, s_{0}\right)=\int_{s_{0}-\Delta s}^{s_{0}+\Delta s} p(s) \exp \left(-2 k^{2} \mu_{s}^{\prime} s D_{B} \tau\right) \mathrm{d} s$,

where $p(s)$ is the normalized distribution of path lengths within $\left[s_{0}-\Delta s s_{0}+\Delta s\right]$. This equation is used for calculating $g_{1}$ if the pathlength distribution can be obtained, e.g., from the MC simulation. Since $s=v \cdot t, v$ is the speed of light in the medium, $t$ is the photon flying time, the pathlength-resolved $g_{1}(\tau, s)$ can be easily converted to the time-resolved $g_{1}(\tau, t)$.

\subsection{Monte Carlo Simulations}

We used the most recently developed MC tool, Mesh-based Monte Carlo (MMC) ${ }^{16}$ to simulate the photon migration inside a realistic human-head model. The human-head model was segmented into four different tissues: skin and skull (SS), cerebral spinal fluid (CSF), gray matter (GM), and white matter (WM). The optical parameters used in the simulations are listed in Table $1 .{ }^{17}$ The wavelength of the illumination light was $830 \mathrm{~nm}$.

Simulations were performed for two cortical regions including the frontal and temporal lobes. The gate opening time was considered from 400 to $1000 \mathrm{ps,} \mathrm{in} 100$ ps steps. The gate width was selected from 50 to 3000 ps. Ten SD distances were used ranging from 0 to $45 \mathrm{~mm}$, in $5 \mathrm{~mm}$ steps. The probes positions for the two regions are shown in Fig. 1. In the MC simulation, the survival weight and partial pathlength in each tissue component were recorded for each emitted photon. The time-resolved diffuse reflectance can thus be calculated for each SD distance. The probing sensitivity of photon to the brain can be determined by the fraction of the total light attributable to the brain (brain = $\mathrm{GM}+\mathrm{WM}$ ). By calculating the ratio of the partial pathlength passing through the brain over the total pathlength of photons collected within a selected gate, we can know the pathlength contribution of the brain for each selected gate.

More specifically, we recorded the pathlength (and partial pathlength) for each emitted photon in MC simulation and obtained the weight for each photon based on the BeerLambert law. Thus once we selected a gate characterized by the opening time $t_{0}$ (denoting the time when the gate starts opening) and width $T$, we knew the weight $\left(W_{d}, d=1,2, . ., M\right)$ for each photon detected and the total weight, $W_{\text {total }}=\sum_{d=1}^{M} W_{d}$, of all detected photon (i.e., $M$ photons) within the gate. Then, we could calculate the relative weight by $W_{i}=W_{d} / W_{\text {total }}$ for

Table 1 Optical properties at $830 \mathrm{~nm}$ used in the Monte Carlo simulations of the realistic human-head model.

\begin{tabular}{lcccc} 
Tissue & $\mu_{a}\left(\mathrm{~mm}^{-1}\right)$ & $\mu_{s}\left(\mathrm{~mm}^{-1}\right)$ & $g$ & $n$ \\
\hline SS & 0.0191 & 6.6 & 0.9 & 1.40 \\
CSF & 0.0026 & 0.1 & 0.9 & 1.40 \\
GM & 0.0186 & 11.1 & 0.9 & 1.40 \\
WM & 0.0186 & 11.1 & 0.9 & 1.40 \\
\hline
\end{tabular}


(a)

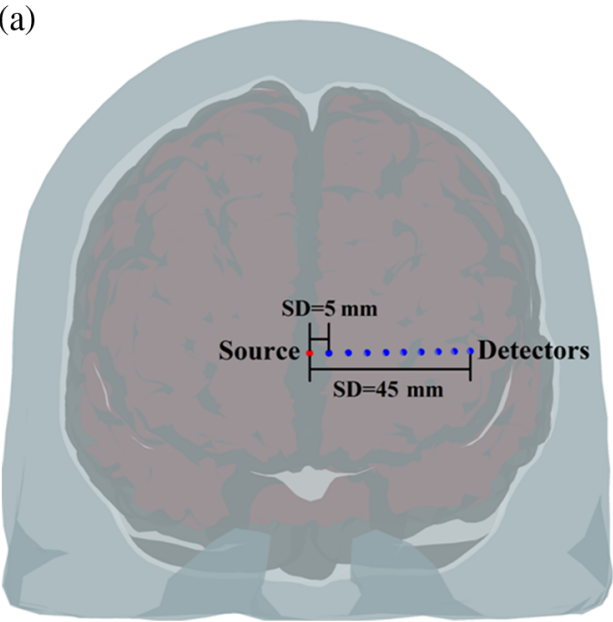

(b)

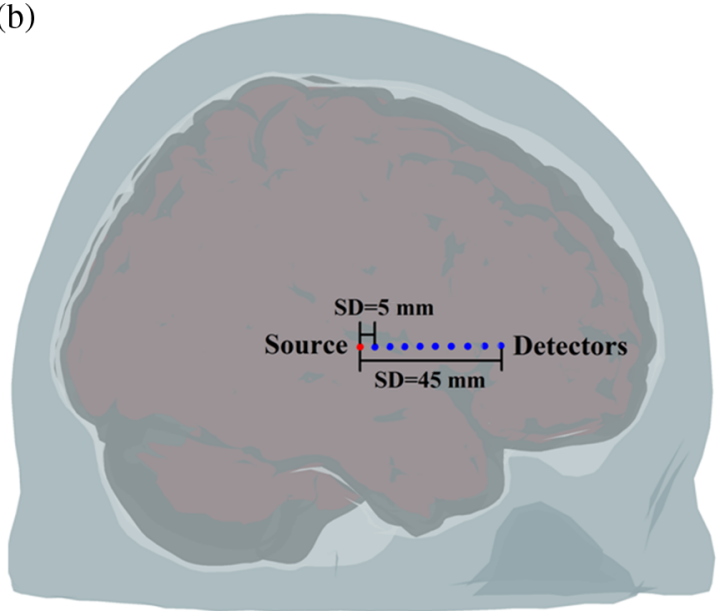

Fig. 1 Locations of the optical probes at the (a) frontal lobe and (b) temporal lobe for the Collin27 model. One source (red point) and 10 detectors (blue points with one overlaid with the red source point) were used in each region with a distance of $5 \mathrm{~mm}$ in between. The light was illuminated perpendicular to the surface of the head. Note that for the simulation on the frontal lobe, although the source was placed over the longitudinal fissure, simulation with other source positions showed that the influence of the fissure was negligibly small.

each photon among all detected photons (i.e., $M$ photons) within the gate. With the known parameters $\mu_{s}^{\prime}, s$, and $D_{B}$ for each layer (component), we performed the summation in Eq. (3) to calculate the time-resolved $g_{1}\left(\tau, t_{0}\right)$ for the selected gate

$g_{1}\left(\tau, t_{0}\right)=\sum_{i=1}^{M} W_{i} \exp \left[-2 k^{2} \tau \sum_{j=1}^{N} \mu_{s}^{\prime(j)} s_{i j} D_{B}^{(j)}\right]$.

The index $j$ denotes each tissue component from 1 to $N(N=4$ here), and $s_{i j}$ is the partial pathlength of the photon $i$ in component $j$. All the MC simulations were performed on a computer running Windows 10 (64 bit) with an Intel Xeon E5-2670 (2.6G) 8 -core processor and the memory of $64 \mathrm{~GB}$. The simulation time was about $14 \mathrm{~h}$ with MMC method for $1.4 \times 10^{9}$ photons in each simulation. In previous similar work of MC simulation, $(1-3) \times 10^{8}$ photons were used, which has been reported to give good photon statistics in medium with optical properties equivalent to biological tissue. ${ }^{16,18}$ The number of launched photons we used was larger than those values reported. We performed eight simulations and found for all SD distances used in this study, the variation of the diffuse reflectance was very small (e.g., $3 \%$ to $5 \%$ ) within the time range, e.g., $<2500$ ps.

\subsection{TD-DCS Simulations}

As mentioned above, TD-DCS is able to differentiate dynamics sampled by different paths in the medium and achieve higher contrast measurement on the change in deep dynamics, such as change on the CBF induced by functional activation. To test this, we mimicked real experimental conditions to perform simulations on the realistic human-head model for the light intensity autocorrelation function $g_{2}$. Two experimental conditions, baseline and stimulation, were considered to investigate the measurement contrast on the change of cortical dynamics. Table 2 shows the diffusion coefficient $D_{B}$ of scatterers in the baseline and stimulation conditions. ${ }^{19,20}$ During the stimulation (or activation), the dynamics in cortex (including GM and $\mathrm{WM}$ ) was assumed to be enhanced by $50 \%$.
Table 2 Brownian diffusion coefficients used in DCS simulations on the realistic human-head model for baseline and stimulation.

\begin{tabular}{lcc} 
Tissue & $\begin{array}{c}D_{B}\left(\mathrm{~mm}^{2} / \mathrm{s}\right) \\
\text { during baseline }\end{array}$ & $\begin{array}{c}D_{B}\left(\mathrm{~mm}^{2} / \mathrm{s}\right) \\
\text { during stimulation }\end{array}$ \\
\hline SS & $1 \times 10^{-6}$ & $1 \times 10^{-6}$ \\
CSF & 0 & 0 \\
GM & $6 \times 10^{-6}$ & $9 \times 10^{-6}$ \\
WM & $2 \times 10^{-6}$ & $3 \times 10^{-6}$ \\
\hline
\end{tabular}

We assumed the average incident power of laser $(830 \mathrm{~nm})$ was $75 \mathrm{~mW}$, and the integration time for $g_{2}$ was $5 \mathrm{~s}$, which barely met the temporal requirement for brain functional detection. In simulated experiments, a single-mode fiber $(5 \mu \mathrm{m}$ in diameter, numerical aperture $\mathrm{NA}=0.22$ ) was used to detect emitted photons. The quantum efficiency of the photon detector (such as avalanche photodiode, APD) was assumed to be $60 \%$. The repetition rate of the pulsed laser was set at $100 \mathrm{MHz}$. The photons detected for each incident pulse for a selected gate (including the gate opening time and width) can be obtained from the MC simulation. By generating a photon sequence with a known count rate and correlation among photons, the $g_{2}$ can be simulated for the baseline and stimulation conditions. ${ }^{21}$

To find out the optimal combinations of SD distance, gate opening time $\left(t_{0}\right)$, and width $(T)$ corresponding to the higher contrast for the change of cortical dynamics, we defined two parameters which we call SNR1 and SNR2, where SNR stands for signal-to-noise ratio evaluated by $\mathrm{SNR}=S / N$. The $\mathrm{SNR}$ was evaluated based on the temporal intensity autocorrelation $g_{2}$ instead of dynamic parameter $D_{B}$. This is because in DCS experiment, $g_{2}$ is the raw data measured directly. From MC simulation, we obtained the pathlength distribution and weight for each path and then calculated the $g_{1}$ by Eq. (3). While $g_{2}$ was simulated based on the correlation of photons (e.g., the decay 
time obtained from the $g_{1}$ ), photon count per sampling time, integration time, and polarization of the light. In the simulation of $g_{2}$, the intercept, i.e., $g_{2}(\tau=0)$ was randomly (or automatically) generated. The first parameter, SNR1, was based on the difference in the decay time $\tau_{0}$ obtained from the fit of $g_{2}$ with a single exponential function $\left[g_{2}(\tau)-1=\beta \exp \left(-\tau / \tau_{0}\right)\right]$. During the fitting, we kept $\beta=0.5$ (for unpolarized light) and searched the decay time $\tau_{0}$ for the best fit. The second parameter SNR2 relied on the difference in $g_{2}$. For SNR1, the signal $S$ was defined as the difference in the decay time $\left[\Delta \tau_{0}=\tau_{0 \text { (baseline) }}-\tau_{0 \text { (stimulation) }}\right]$ between theoretical (or noisefree) $g_{2}$ for the baseline and stimulation. The noise $N$ was defined as the standard deviation of the $\Delta \tau_{0}$ estimated from many (e.g., 100 times) simulations on $g_{2}$. SNR2 relied on the difference in $g_{2}$ between the baseline and stimulation. The maximal difference in theoretical $g_{2}$ [i.e., $\Delta g_{2}\left(\tau=\tau_{m}\right)$ ] was considered as the signal $S$, and the corresponding (e.g., at the same lag time $\tau_{m}$ ) standard deviation of $\Delta g_{2}$ estimated from many (i.e., 100 times) simulations on $g_{2}$ as the noise $N$. The $\tau_{m}$ was the lag time $\tau$ when the difference in theoretical $g_{2}$ between the baseline and stimulation was maximal.

\section{Results}

\subsection{Diffuse Reflectance and Pathlength Distribution in the Brain}

From the MC simulations, the time-resolved diffuse reflectance at the head surface can be obtained for each SD distance. Figure 2 shows the time-resolved diffuse reflectance (photons $/ \mathrm{mm}^{2} \mathrm{ps}$ ) for each incident photon for $10 \mathrm{SD}$ distances at the frontal lobe [Fig. 2(a)] and temporal lobe [Fig. 2(b)]. As expected, the reflectance decreases as the time increases, but increases as the SD distance decreases. Late arriving photons imply the photons experience longer paths, thus probe deeper in the brain. However, the number of later arriving photons decreases dramatically as the time increases (or pathlength increases). Thus in real experiment with a limited illumination power, the balance between the detection sensitivity (to the brain) and the detected light intensity should be considered. The photon number for a selected gate can be obtained from the diffuse reflectance, which is used for simulating the intensity autocorrelation function $g_{2}$ with a certain illumination power.
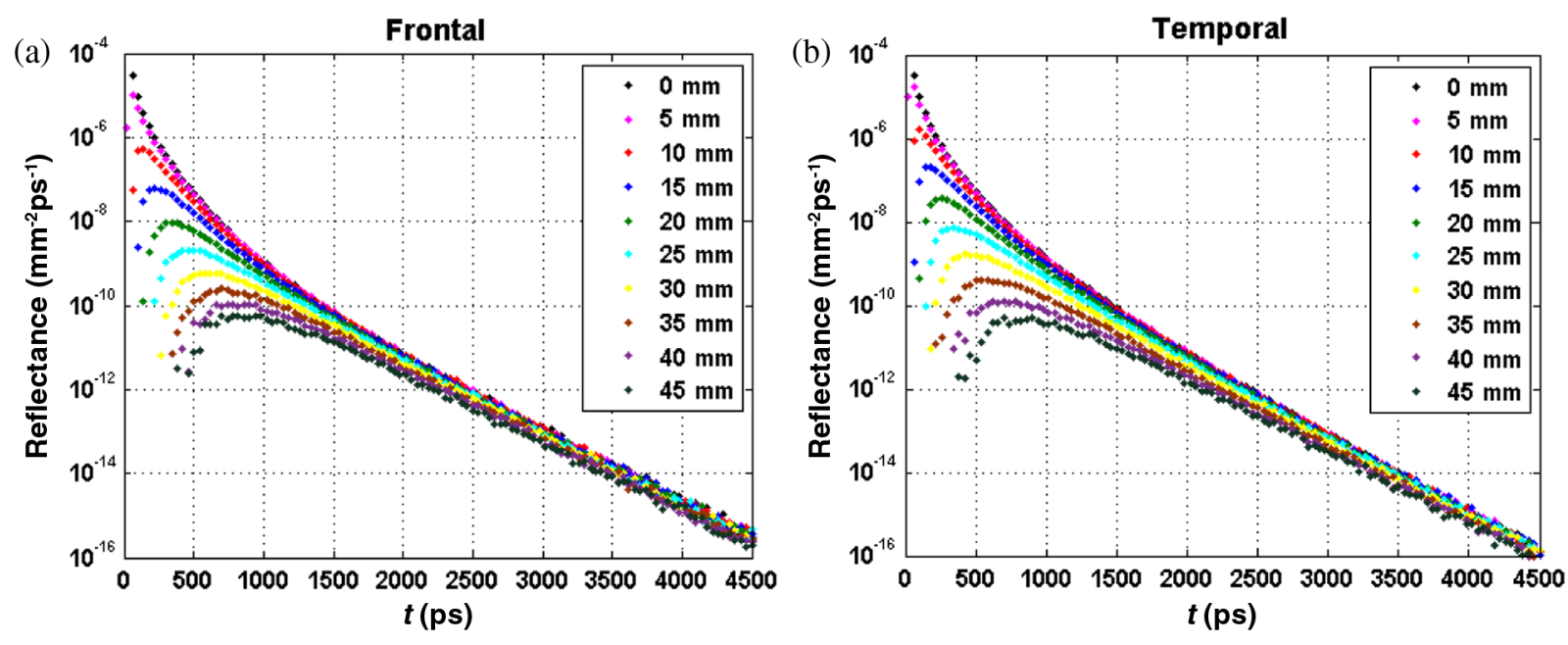

Fig. 2 The diffuse reflectance as a function of time at $10 \mathrm{SD}$ distances from 0 to $45 \mathrm{~mm}$ for the (a) frontal lobe and (b) temporal lobe.
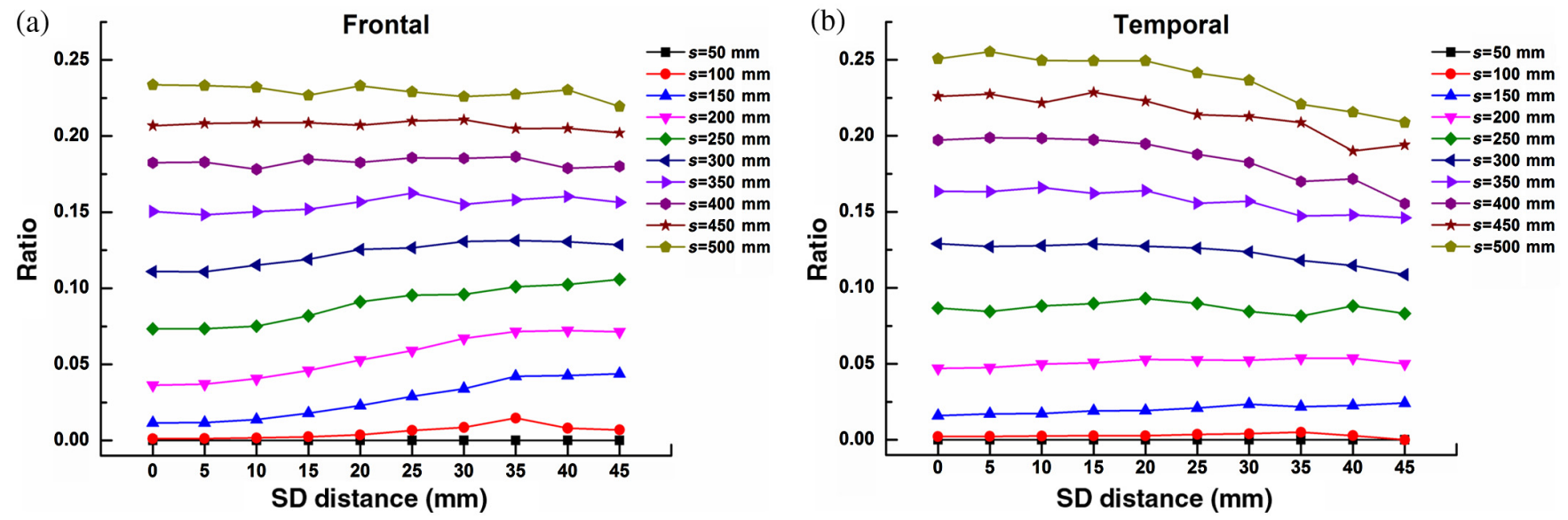

Fig. 3 Ratios of the partial pathlength in the brain (brain = GM + WM) to the total pathlength for 10 selected pathlengths in the range 50 to $500 \mathrm{~mm}$ and $10 \mathrm{SD}$ distances in the range of 0 to $45 \mathrm{~mm}$ in the (a) frontal lobe and (b) temporal lobe. 
The partial pathlength in each tissue component can be also obtained from the MC simulation. For a certain pathlength, the ratio of the partial pathlength passing through the brain over the total pathlength reveals the contribution from the brain to the measured $g_{2}$. Figure 3 shows the partial pathlength ratios of brain for 10 pathlengths in the range 50 to $500 \mathrm{~mm}$ in steps of $50 \mathrm{~mm}$ and $10 \mathrm{SD}$ distances in the range 0 to $45 \mathrm{~mm}$ in steps of $5 \mathrm{~mm}$. For both frontal and temporal regions, the ratios of the partial pathlength in the brain at short pathlengths such as 50 and $100 \mathrm{~mm}$ are almost zero for nearly all the 10 SD distances, which means the shortest pathlength of photons passing through the brain is longer than $100 \mathrm{~mm}$. In other words, photons with these short pathlengths do not provide information about the brain. Therefore, in searching optimal parameter for the gate opening time $\left(t_{0}\right)$, we excluded the early arriving photons and started from $400 \mathrm{ps}$, corresponding to about
$86 \mathrm{~mm}$ of photon traveling distance in the medium. Because only photons with arriving time larger than 400 ps are possible to interrogate the brain, which is also demonstrated by the observation that with the increase of the pathlength, the partial pathlength ratio of brain increases.

\subsection{Optimization for TD-DCS Measurement}

The balance between the photon arriving time (or pathlength) and associated light intensity should be considered in TDDCS measurements. Typically, using the late photons can theoretically increase the probing sensitivity to the brain, but the number of these late photons is generally small. Lower intensity of light gives rise to larger noise level on $g_{2}$. Herein, we use the SNR to evaluate the trade-off between the time (or pathlength) and the detected light intensity, and then further find out optimal
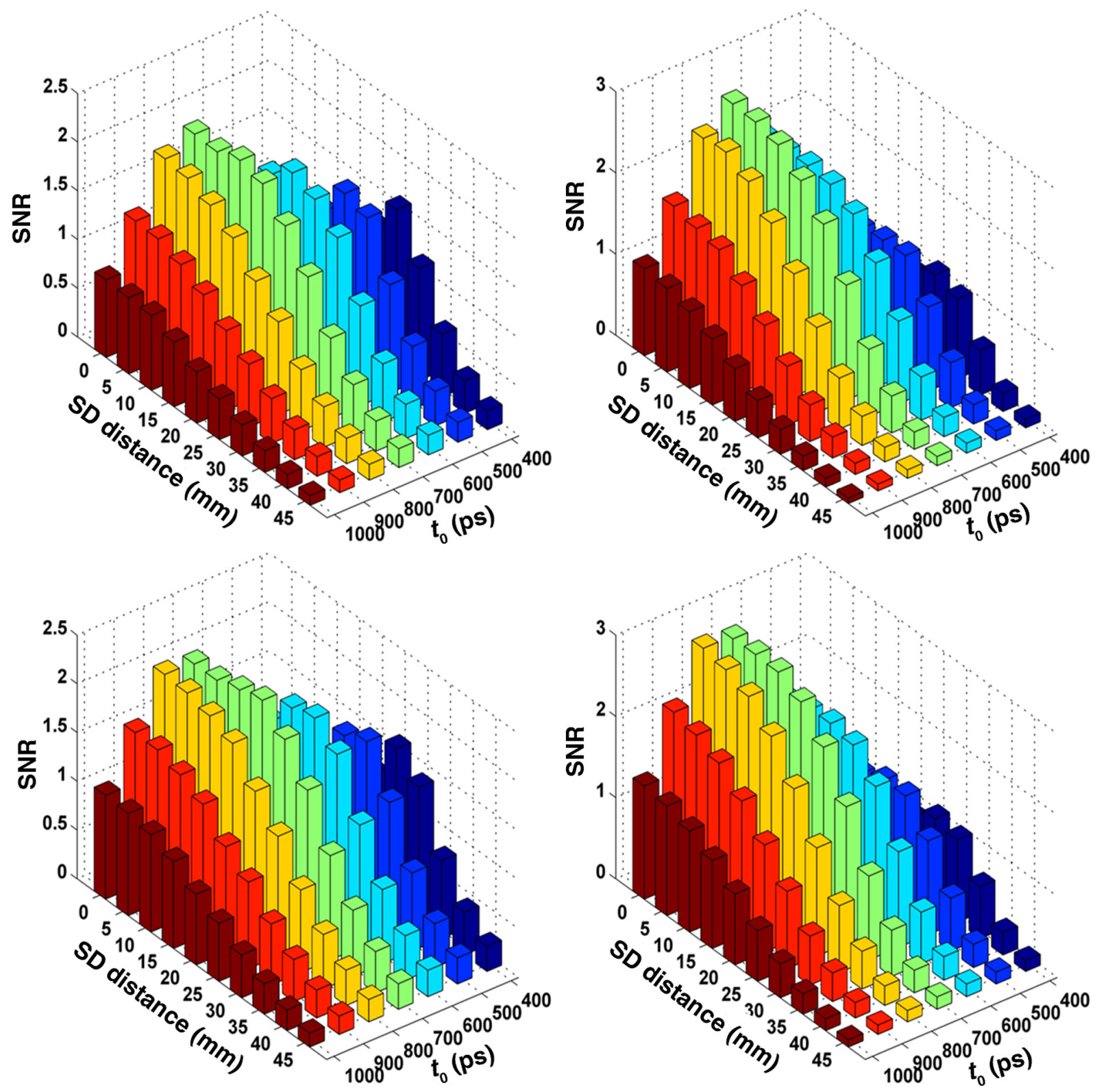

Fig. 4 The SNR values calculated based on $\Delta \tau_{0}$ (upper row) and on $\Delta g_{2}$ (bottom row) for seven gate opening time $t_{0}$ from 400 to $1000 \mathrm{ps}$ in steps of $100 \mathrm{ps}$, and $10 \mathrm{SD}$ distances in the range of 0 to $45 \mathrm{~mm}$ in steps of $5 \mathrm{~mm}$ for the frontal lobe (left column) and temporal lobe (right column). The gate width $T$ is 800 ps. 
combinations of SD distance and the gate by which the higher contrast (i.e., larger SNR) measurement on the change of cortical dynamics can be achieved. Three parameters were investigated including the SD distance, gate opening time $\left(t_{0}\right)$, and the gate width $(T)$. Simulations show the optimal combinations of these three parameters are SD distance ranging from 0 to $15 \mathrm{~mm}$, gate opening time at 700 to $800 \mathrm{ps}$, and the width of gate larger than 800 ps. Figure 4 shows the SNRs calculated for seven gate opening time $t_{0}$ from 400 to $1000 \mathrm{ps}$ in steps of $100 \mathrm{ps}$, and 10 SD distances in the range of 0 to $45 \mathrm{~mm}$ in steps of $5 \mathrm{~mm}$, with the gate width of $800 \mathrm{ps}$. In the both cortical regions, the two SNR approaches give similar results: the higher SNR values are shown in the combinations of the gate opening time $t_{0}$ at 700 to $800 \mathrm{ps}$ and SD distances of 0 to $15 \mathrm{~mm}$. Figure 5 shows the SNRs dependence on the gate width and gate opening time for the SD distance $=10 \mathrm{~mm}$.

Varying the gate width induces change in the photon count, thus affects noise level on $g_{2}$ and the SNRs, which is shown in Fig. 6. For the late gate with the opening time $\left(t_{0}\right)$ larger than
$400 \mathrm{ps}$, the SNRs increase with respect to the gate width $(T)$, but nearly saturates after $T=800$ ps. Late arriving photons probe deeper in the brain; however, the number of such photons with arriving time larger than, e.g., $700\left(t_{0}\right)+800(T)$ ps $=1500$ ps, is rather small (see Fig. 2), thus these late photons make negligible contribution to the SNRs as observed no further increase after $T=800 \mathrm{ps}$.

To show the difference between the baseline and stimulation on the "measured" $g_{2}$, three examples of $g_{2}$ for the frontal cortex are presented in Fig. 7, including a combination within the optimal range, a combination beyond the optimal range, and $g_{2}$ measured with CW-DCS. All are under the same condition of the illumination light power $(75 \mathrm{~mW})$ and integration time $(5 \mathrm{~s})$. For a combination within the optimal range: SDdistance $=0 \mathrm{~mm}, t_{0}=800 \mathrm{ps}$, and $T=1000 \mathrm{ps}$ [Fig. 7(a)], the difference on the time-resolved $g_{2}$ between the baseline and stimulation can be visually differentiated. However, for a combination far beyond the optimal range: SD distance $=$ $0 \mathrm{~mm}, t_{0}=800 \mathrm{ps}$, and $T=100 \mathrm{ps}$ [Fig. 7(b)], the two curves
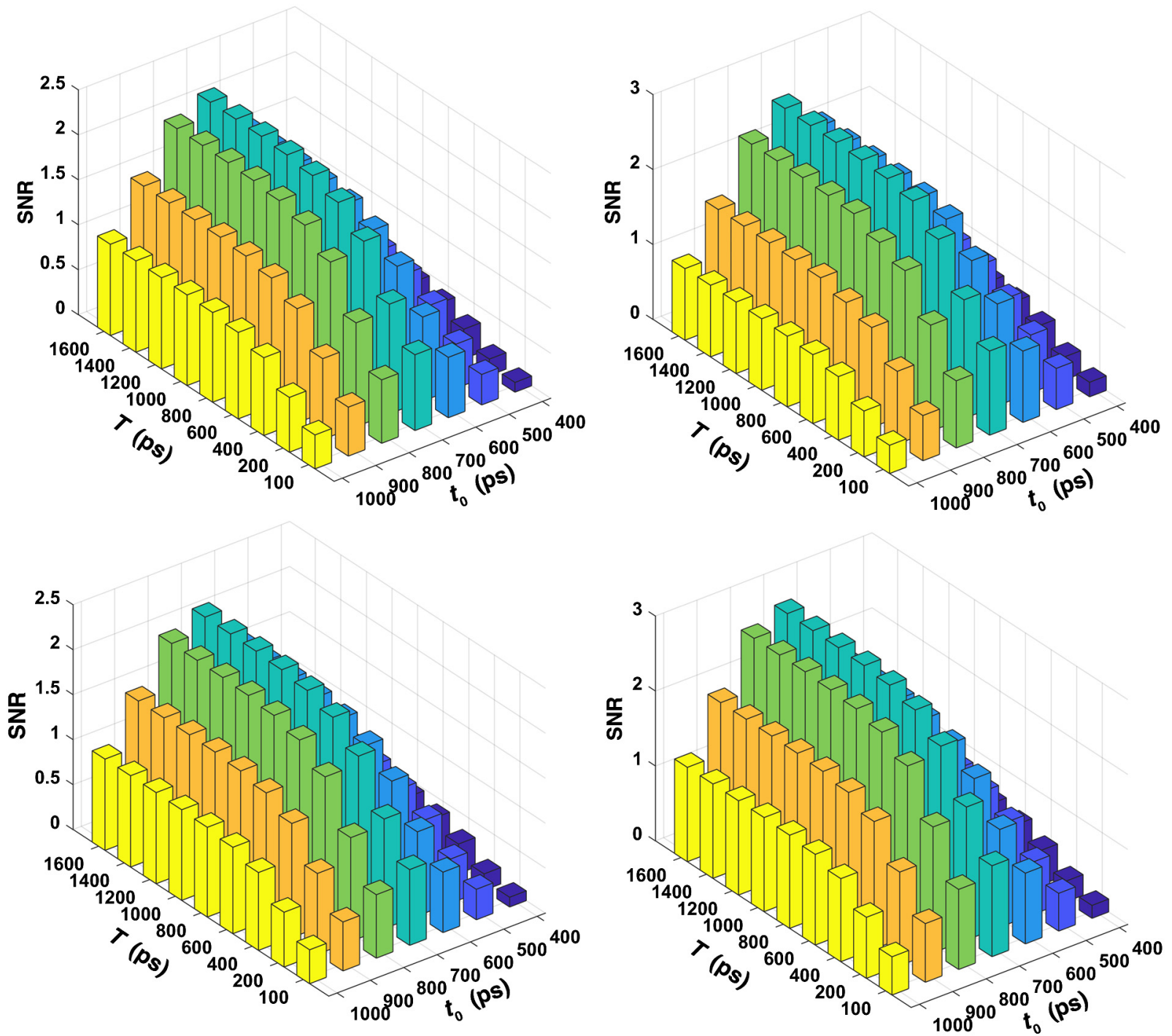

Fig. 5 The SNR values calculated based on $\Delta \tau_{0}$ (upper row) and on $\Delta g_{2}$ (bottom row) for seven gate opening time $t_{0}$ from 400 to $1000 \mathrm{ps}$, and nine gate width $T$ from 100 to $1600 \mathrm{ps}$ for the frontal lobe (left column) and temporal lobe (right column). The SD distance is $10 \mathrm{~mm}$. 
(a)

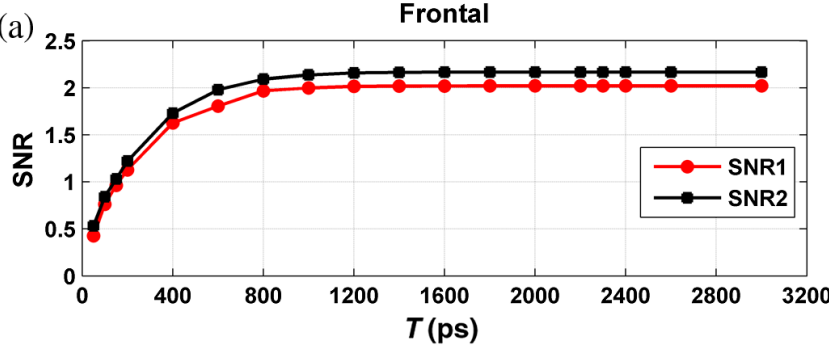

(b)

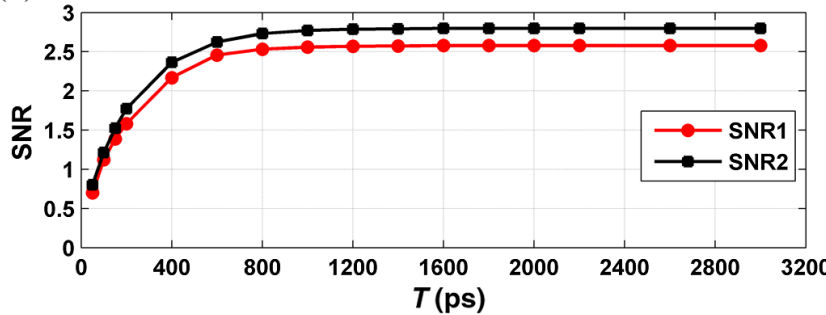

Fig. 6 The SNR as a function of gate width $(T)$ for (a) frontal lobe and (b) temporal lobe. The gate opening time $t_{0}=700 \mathrm{ps}$, $\mathrm{SD}$ distance $=10 \mathrm{~mm}$. It might be noted that the effect of gate width $(T)$ on SNRs for the other combinations are similar to the example presented here.

are completely indistinguishable. For the CW-DCS measurement with SD distance $=30 \mathrm{~mm}$ [Fig. 7(c)], it is hard to visually identify the difference between the baseline and stimulation.

\section{Discussions}

Under the condition of a limited incident light power (e.g., $75 \mathrm{~mW}$ ) and 5-s integration time for the intensity autocorrelation function $g_{2}$, we used a simulation approach to investigate the relationship between the sensitivity of TD-DCS measurement on the cortical dynamics and the combination of SD distance and the gate (including gate opening time and gate width). In the simulation we used $75-\mathrm{mW}$ average power, which was, on one hand, based on our previous experience in CW-DCS measurement on the human head, ${ }^{8}$ on the other hand, it was feasible to ensure the safety limit by slightly expanding the illumination area on the scalp. As reported in the ANSI Standard Z136.1-1993 Table, ${ }^{9}$ the maximum permissible exposure of skin to laser radiation is between 2.6 and $4 \mathrm{~mW} / \mathrm{mm}^{2}$ in the 760 to $850 \mathrm{~nm}$ range. Thus, the incident power of $75 \mathrm{~mW}$ is possible as long as the illumination area is expanded to $\sim 20 \mathrm{~mm}^{2}$ (e.g., a circular speckle with a radius of $\sim 2.5 \mathrm{~mm}$ ). The number of photons injected into the scalp per picosecond can be estimated from the incident power and the wavelength. Since the time-resolved diffuse reflectance (i.e., photons $/ \mathrm{mm}^{2}$ ps for each injected photon) can be calculated from the MC simulation, with 75-mW incident power, the number of photons emitted at a certain time (within a time widow) and for an SD distance can be obtained. This number of emitted photons is used for simulating $g_{2}$ according to the approach proposed in Ref. 21, in which the integration time is also a necessary parameter. For functional monitoring of human brain, the integration time is usually the lower limit for the sampling time of the CBF. The $5 \mathrm{~s}$ we used is certainly not very good for the temporal resolution of measuring $\mathrm{CBF}$, but still acceptable for the slow hemodynamic signal. To improve the temporal resolution, and at the same time keep the same SNR, higher incident power must be used, which is however limited by the safety regulation.

In simulations with the human-head model, we mimicked the actual experimental condition (including the incident power and all related features of optoelectronic detection) and calculated the light intensity autocorrelation function $g_{2}$ for the baseline and stimulation. By simulations with a variety of combinations of SD distance, gate opening time, and gate width, we found that with the combinations of SD distance ranging from 0 to $15 \mathrm{~mm}$, gate opening time at 700 to $800 \mathrm{ps}$, and the gate width larger than 800 ps, TD-DCS was able to achieve higher contrast measurement on the change of cortical dynamics. Sutin et al. ${ }^{10}$ have demonstrated that the measurement sensitivity for the CBF can be improved by analyzing photons with longer pathlengths. Photons with longer pathlengths interrogate deeper tissue, thus carrying more information on the brain, which is also revealed by the partial pathlength ratio of the brain (see Fig. 3). However, with the increase of the photon pathlength (or photon arriving time), the number of detected photons decreases dramatically (see Fig. 2), which results in large noise level on the $g_{2}$, eventually deteriorating the measurement sensitivity for the brain. Therefore, there exists an optimal range for those three experimental parameters resulting from a
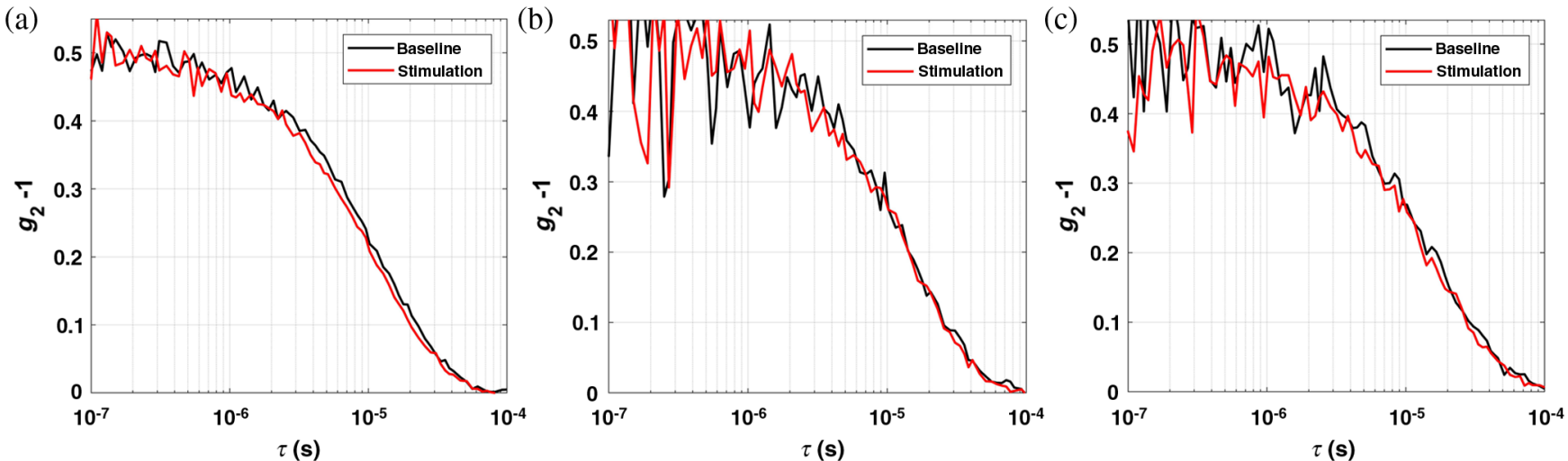

Fig. 7 Simulated TD and CW $g_{2}$ for the baseline (black line) and stimulation (red line) over the frontal region. (a) An optimal combination of SD distance $=0 \mathrm{~mm}, t_{0}=800 \mathrm{ps}$, and $T=1000 \mathrm{ps}$, with SNR1 $=2.39$, SNR2 $=2.64$. (b) A combination far beyond the optimal range with SD distance $=0 \mathrm{~mm}$, $t_{0}=800 \mathrm{ps}$, and $T=100 \mathrm{ps}$, with SNR1 $=0.79, \mathrm{SNR} 2=0.67$. (c) The CW $g_{2}$ at SD distance $=$ $30 \mathrm{~mm}$, with SNR1 $=1.33, \mathrm{SNR} 2=1.49$. 
compromise between the photon pathlength and photon count detected.

Different from CW-DCS setup using a CW laser source, TD-DCS utilizes a pulse laser source. For any pulse laser, the coherence length of light is limited by the principle of uncertainty, e.g., for pulse duration of $150 \mathrm{ps}$, the upper limit value of the coherence length is about $40 \mathrm{~cm}$. In practice, the value of the coherence length of a pulse laser is generally less than the upper limit. When the gate width $T=800 \mathrm{ps}$, the difference in pathlength between the shortest and longest is $v \cdot T \approx 16 \mathrm{~cm}$ ( $v$ is the speed of light in the tissue which is about $2 \times 10^{10} \mathrm{~cm} / \mathrm{s}$ ). To ensure the all photons detected within the gate are correlated, the differences between all pathlengths should be smaller than the coherence length. Otherwise, the intercept $\beta$ will decrease due to the incoherence of photons detected, deteriorating $g_{2}$ measurement on the other hand. In TD-DCS measurements, the coherence length of the pulsed laser used may be different for different setups. In searching optimal parameter of gate width $(T)$, we did not consider the influence of the coherence length of the light. The result shows when the gate width reaches $800 \mathrm{ps}$, the two SNRs nearly reach their maxima. Therefore, as long as the light coherence length is longer than $16 \mathrm{~cm}$, our conclusion holds.

A limitation of this study is the fact that we have not considered the effect of the nonideality of the measurement system, typically described by the instrument response function (IRF) and obtained by recording the photon pathlength distribution when facing the injection and collection fibers. The fullwidth-at-half-maximum (FWHM) is normally used as a synthetic descriptor for the IRF. Typical values for the FWHM of time-domain near-infrared spectroscopy (TD-NIRS) systems are in the range of 35 to $600 \mathrm{ps}^{22}$ When the FWHM is less than $250 \mathrm{ps}$, its influence on time-gating methods can be negligible. ${ }^{23}$ For TD-DCS, our simulation study has shown that the impact is small for FWHM of IRF $<125$ ps. In the few recent papers on TD-DCS, ${ }^{10,11}$ the FWHM was narrower than or equal to $100 \mathrm{ps}$, which would have nearly no influence on our conclusion. The main expected implication of using a broad IRF will be a decreased ability of the system to discriminate early and late photons. We can in fact consider the FWHM of the IRF as an indication of the error related to the timing of detected photons. As a consequence, we expect a decreased contrast to deep changes in both hemoglobin concentration and blood flow. A further effect will be an error in the estimate of the optical properties of the tissue when fitting the distribution of photon timeof-flight with a model for photon migration. This will have an impact on the estimate of the blood flow). Similarly, a limited SNR of late photons would affect the ability of the system to discriminate deep changes, since the distribution of photon time-of-flight will be richer in early photon, but lower in late photons. In this work, we have not included the detailed study on the effect of IRF, since it is beyond the scope of this work.

In contrast to NIRS, DCS generally requires a single-mode fiber (with a small core diameter) for light detection. In many practical measurements for human head, under the safety limit of incident power, the light intensity detected and transmitted through a single-mode fiber is generally low, easily resulting in large noise level on the measured $g_{2}$. In CW-DCS, utilizing a shorter SD distance (e.g., $<25 \mathrm{~mm}$ ) may lead to higher detected light intensity, but reduce the sensitivity of detection for deeper dynamics. However, in TD-DCS, we found that the relatively shorter $(\leq 15 \mathrm{~mm})$ or even null SD distance can also achieve good sensitivity to deep dynamics as long as an optimal gate was selected, which is very similar to the null SD distance detection in TD-NIRS. ${ }^{24} \mathrm{~A}$ concern of using null (or very short) SD distance in an actual experiment is that it may induce saturation of photon detector (such as APD) thus cause problem. To deal with this problem, Pifferi et al. ${ }^{25}$ proposed a gating detection approach, in which an APD was operated in time-gated mode to prevent detection of the early photons and enhance contribution of late photons.

\section{Conclusions}

By using Monte Carlo simulations on a realistic human-head model, under the condition of limited illumination power $(75 \mathrm{~mW})$, we have investigated the relationship between the sensitivity of probing deeper dynamics and combinations of SD distance, gate opening time, and gate width in TD-DCS. We found that higher contrast measurement on the changes in deeper dynamics such as CBF induced by functional activation can be achieved by selecting the optimal SD distance and gate combination. In TD-DCS measurement for human brain function, to achieve higher detection contrast to the $\mathrm{CBF}$, the combination of SD distance of 0 to $15 \mathrm{~mm}$, gate opening time at 700 to $800 \mathrm{ps,}$ and gate width of $800 \mathrm{ps}$ are optimal options. These results may be helpful in future TD-DCS experiments aiming at detecting human brain functional activity.

\section{Disclosures}

The authors of this paper report no relevant financial interests or other potential conflicts of interest to disclose.

\section{Acknowledgments}

This work was supported by the Guangdong Provincial Key Laboratory of Optical Information Materials and Technology (Grant No. 2017B030301007), Guangdong Science and Technology Program (Grant No. 2017A010101023), and the National Natural Science Foundation of China (NSFC) (Grant No. 81771876).

\section{References}

1. G. Maret and P. E. Wolf, "Multiple light scattering from disordered media: the effect of Brownian motion of scatterers," Z. Phys. B: Condens. Matter 65, 409-413 (1987).

2. D. J. Pine et al., "Diffusing-wave spectroscopy," Phys. Rev. Lett. 60, 1134-1137 (1988).

3. D. J. Pine et al., "Diffusing-wavespectroscopy: dynamic light scattering in the multiple scattering limit," J. Phys. 51, 2101-2127 (1990).

4. D. A. Boas, L. E. Campbell, and A. G. Yodh, "Scattering and imaging with diffusing temporal field correlations," Phys. Rev. Lett. 75, 18551858 (1995).

5. D. A. Boas and A. G. Yodh, "Spatially varying dynamical properties of turbid media probed with diffusing temporal light correlation," J. Opt. Soc. Am. A 14, 192-215 (1997).

6. C. Zhou, "In-vivo optical imaging and spectroscopy of cerebral hemodynamics," PhD Dissertation, University of Pennsylvania (2007).

7. R. C. Mesquita et al., "Direct measurement of tissue blood flow and metabolism with diffuse optics," Philos. Trans. R. Soc. A: Math. Phys. Eng. Sci. 369(1955), 4390-4406 (2011).

8. J. Li et al., "Noninvasive detection of functional brain activity with nearinfrared diffusing-wave spectroscopy," J. Biomed. Opt. 10(4), 044002 (2005).

9. Standard, A. N. S. I., American National Standard for the Safe Use of Lasers, Z136. 1-1993, American National Standards Institute Inc., New York (1993).

10. J. Sutin et al., "Time-domain diffuse correlation spectroscopy," Optica 3(9), 1006-1013 (2016) 
11. M. Pagliazzi et al., "Time domain diffuse correlation spectroscopy with a high coherence pulsed source: in vivo and phantom results," Biomed. Opt. Express 8, 5311-5325 (2017).

12. J. Li et al., "Analytical models for time-domain diffuse correlation spectroscopy for multi-layer and heterogeneous turbid media," Biomed. Opt. Express 8, 5518-5532 (2017).

13. D. L. Collins et al., "Design and construction of a realistic digital brain phantom," IEEE Trans. Med. Imaging 17(3), 463-468 (1998).

14. P. A. Lemieux and D. J. Durian, "Investigating non-Gaussian scattering processes by using nth-order intensity correlation functions," J. Opt. Soc. Am. A 16, 1651 (1999).

15. T. Durduran et al., "Diffuse optics for tissue monitoring and tomography," Rep. Prog. Phys. 73, 076701 (2010).

16. Q. Fang, "Mesh-based Monte Carlo method using fast ray-tracing in Plücker coordinates," Biomed. Opt. Express 1(1), 165-175 (2010).

17. G. Strangman, M. A. Franceschini, and D. A. Boas, "Factors affecting the accuracy of near-infrared spectroscopy concentration calculations for focal changes in oxygenation parameters," Neurolmage 18(4), 865-879 (2003)

18. L. Gagnon et al., "Investigation of diffuse correlation spectroscopy in multi-layered media including the human head," Opt. Express 16(20), 15514-15530 (2008).
19. J. Selb et al., "Sensitivity of near-infrared spectroscopy and diffuse correlation spectroscopy to brain hemodynamics: simulations and experimental findings during hypercapnia," Neurophotonics 1(1), 015005 (2014).

20. S. C. Ramsay et al., "Changes in global cerebral blood flow in humans: effect on regional cerebral blood flow during a neural activation task," J. Physiol. 471(1), 521-534 (1993).

21. F. Ferri and D. Magatti, "Hardware simulator for photon correlation spectroscopy," Rev. Sci. Instrum. 74(10), 4273-4279 (2003).

22. A. Torricelli et al., "Time domain functional NIRS imaging for human brain mapping," NeuroImage 85, 28-50 (2014).

23. L. Zucchelli et al., "Method for the discrimination of superficial and deep absorption variations by time domain fNIRS," Biomed. Opt. Express 4(12), 2893-2910 (2013).

24. A. Torricelli et al., "Time-resolved reflectance at null source-detector separation: improving contrast and resolution in diffuse optical imaging," Phys. Rev. Lett. 95, 2-5 (2005).

25. A. Pifferi et al., "Time-resolved diffuse reflectance using small sourcedetector separation and fast single-photon gating," Phys. Rev. Lett. 100, 138101 (2008).

Biographies for the authors are not available. 